\title{
Automated Pulse-Based Diagnosis: Role of TIM Diagnostic Features
}

\author{
Rajani R. Joshi \\ Department of Mathematics, Indian Institute of Technology Bombay, Powai, India \\ Email: rrj@math.iitb.ac.in
}

Received 3 June 2014; revised 18 July 2014; accepted 30 July 2014

Copyright (C) 2014 by author and Scientific Research Publishing Inc. This work is licensed under the Creative Commons Attribution International License (CC BY). http://creativecommons.org/licenses/by/4.0/

c) (7) Open Access

\begin{abstract}
Emanated from the idea of reinvestigating ancient medical system of Ayurveda-Traditional Indian Medicine (TIM), our recent study had shown significant applications of analysis of arterial pulse waveforms for non-invasive diagnosis of cardiovascular functions. Here we present results of further investigations analyzing the relation of pulse-characteristics with some clinical and pathological parameters and other features that are of diagnostic importance in Ayurveda.
\end{abstract}

\section{Keywords}

Pulse-Based Diagnosis, Spectral Parameters, TIM (Traditional Indian Medicine) Diagnostic Features, Statistical Analysis

\section{Introduction}

Pulse-examination for diagnosis/prognosis is an important component of traditional Chinese and Indian medical system. Considering that the traditional method of pulse-examination relies upon sensation of the pulse by the doctor, standardization and validation by modern scientific approaches poses formidable challenge in reviving this ancient non-invasive and comprehensive method of diagnosis/prognosis of diseases and psychosomatic disorders. Instrumentation based standardized techniques of pulse-signal recording offer feasible solution in this regard. Digitization of the signal makes room for wide-range of computational analysis and modeling and validation of this method. Our recently reported and tested algorithm of efficient filtering of noise and base-line drift made significant contribution in augmenting this potential [1]. While most other studies reported since last few decades have focused mainly on Heart Rate Variability (HRV) studies, only some, including ours have investigated wider applications.

In our most recent paper we had given a review of important approaches that re-establish and provide novel applications of the ancient Indian and Chinese techniques of diagnosis/prognosis using pulse-examination [2]. In 
view of our earlier work on Ayurvedic (TIM) concepts and computer simulations of pulse waveforms [3]-[5], we had developed a computational algorithm for automatic detection of specific types of morphological patterns in radial arterial pulse waveform using Fisher's Ratio [6] and some geometrical parameters. Validation of this state-of-the-art method on a large sample had shown high accuracy of prediction. We had also analyzed pulse morphology variation (with respect to time) using this method and discussed characteristic results for certain diseased conditions/disorders [2].

In the present paper we consider further study on analysis of spectral parameters of the radial arterial pulse (hereafter mostly referred as pulse) and certain clinical, physiological and pathological features that are crucial to Ayurvedic method of diagnosis/prognosis of ailments/disorders of the mind-body system.

The features and data-set are described in the next Section. Analysis and results are presented in Section 3. Significance of results and scope are highlighted in the last Section.

\section{Features \& Data Set}

The dataset used in our earlier study on pulse morphology variation [2] consisted of the digital IPG signals of (radial arterial) pulse of adult Indians that were provided by our collaborators at BARC, Mumbai and CDAC, Pune. The final sample after filtering of baseline wandering and other noises consisted of pulse-records of 170 Females and 265 Male volunteers. The age of subjects in this sample varied between 20 and 75 years. Those within a comparable age-group, e.g. 20 - 25 or 65 - 75 years, etc., had comparable body-mass-index.

For nearly $65 \%$ of this sample there also were observations provided for all of the following features.

TIM Diagnostic Features: Age (years), Body-weight (kg.), BP (Diastolic, Systolic), Pulse-rate (per min.), Bala, Guna-1, Guna-2, Guna-3, Depth, Doshas.

The features Bala, etc., are specific to Ayurvedic pulse-examination.

Bala indicates perceived force of artery. It has two categories: Balwaan - fullness of artery (pulse coming up with force hence easily palpable); or Ksheen - weakness or diminished flow of artery (pulse needs deep palpation to be felt by the Physician).

Gunas indicate three specific characters of pulse as sensed by the Ayurvedic Physicians. Each has two categories. Guna-1: sthool (broadness usually accompanied by heaviness) or sooksham (subtlety, does not rise against the entire midst of palpating surface of fingertips); Guna-2: guru (heaviness in movement) or laghu (lightness in movement); Guna-3: mridu (soft in touch) or kathin (hard in touch).

Depth identifies superficial or deep status of the pulse: uttaan (palpable easily upon superficial touch) or gambheer (not palpable easily, palpable upon deep touch).

Doshas in Ayurvedic terminology are supposed to indicate natural tendency (Prakrati) of the functional (physiological and biochemical including metabolic) state of the mind-body system [2] [7]-[9]. These are identified in terms of-Vaat (indicative of air/gaseous element); Pitt (indicative of fire element); and Kaph (indicative of water element). Excess or perturbation of either of these indicates pathological conditions. Therefore doshas are also used as pathological parameters in studies of TIM.

Hereafter we will refer the above-described TIM diagnostic features simply as "Features". In the data given to us, the features Bala and Depth were described as categorical so, as per standard practice in statistical quantitative representation, each was converted into two-dimensional variables: namely balwaan $=(0,1)$; ksheen $=(1$, $0) ; \&$ uttaan $=(0,1)$; gambheer $=(1,0)$. Other qualitative features were provided in the data set as scaled integer weights on a scale 0 to 6 assigned by the Ayurvedic Physicians.

\section{Analysis \& Results}

We had carried out multivariate hierarchical clustering of the given data on above-described features. The results showed good relevance of the features in terms of distinct clusters of different health conditions and disease types [10].

\subsection{Significant Findings of Principal Component Analysis}

For further insight into the covariance structure of the features in different classes of healthy and diseases cases, we have also conducted Principal Component Analysis [11]. Computational experiments for this purpose were carried out using SAS software to identify which features contribute the most to the structure of covariance matrices, hence in the overall variation and description of the data in different classes of the subjects/patients. 
Following results were found in terms of loadings for first eight principal components, which explained nearly $92 \%$ of the variation in the data.

Features measuring pulse properties and doshas were found most important factors in representing the variation in data set. Interestingly, Bala $=(0,1)$ and Depth $=(0,1)$ depth were dominating in this respect among the males as well as females. The features BP, Age and body-weight were found to play above-average role in this respect. However Guna-2 and Pitt were dominant features only in Males and Bala $=(1,0)$ was important in Females. Vaat and Kaph were found to have comparable impact on both males and females.

Disease-wise Roles: Bala, and Depth and Vaat were found prominent in distinguishing the subgroup of patients suffering from Hypertension (HT) and/or Ischemic Heart Disease (IHD) from other subjects. Guna-2 had partially significant role in this respect. In case of Diabetes Mellitus (DM), the features Bala, Depth, and body-weight were prominent. Though, less prominent, Guna-1 \& Guna-2 also had significant role in classifying DM from others.

\subsection{Relation of Features with Pulse Spectral Analysis}

Further relevance of the features with respect to pulse waveform was found in terms of Spectral Analysis of the pulse signal records. Plots of power spectral density (PSD) of pulse-signals were found to have distinct patterns in certain diseases [9]. Interestingly, with demarcation of signal frequency ranges as Low Frequency (LF): 0 to $0.1 \mathrm{~Hz}$, Medium Frequency (MF): 0.1 to $0.25 \mathrm{~Hz}$, High Frequency (HF): 0.25 to $0.5 \mathrm{~Hz}$, we found some significant results on prominence of different peaks among subjects with Vaat, Pitt, and Kaph dominance (e.g. Figure 1).

Accuracy of PSD-based estimation of Vaat, Pitt, and Kaph levels vis-à-vis the integer-weights assigned by the experts for these features of doshas, was found about $72 \%$ on an average.

Harmonic analysis also led to significant inference rules, with statistical confidence above $90 \%$, in terms the ratio (technically called the $5^{\text {th }} \mathrm{K}$-ratio; denoted) of power energy ratio of the first five harmonics. These rules provide potential applications for non-invasive and quick diagnosis of cardio-vascular risks, if any [10].

\subsubsection{Significant Relation with Fisher's Ratio}

Fisher's Ratio (FR) is a likelihood ratio of cumulative eperiodogram of a signal (arterial pulse signal in our study). Our recently reported research had shown significant application of FR in-(i) classifying the abnormal and normal morphology-patterns in any selected time-interval of the record; (ii) accurate detection of morphological variations over time that were found to have important role in diagnosis/prognosis of cardiac disorders [2].

In view of these findings, the main focus of our present study is to analyze the relationship between the FR and the TIM Diagnostic Features.

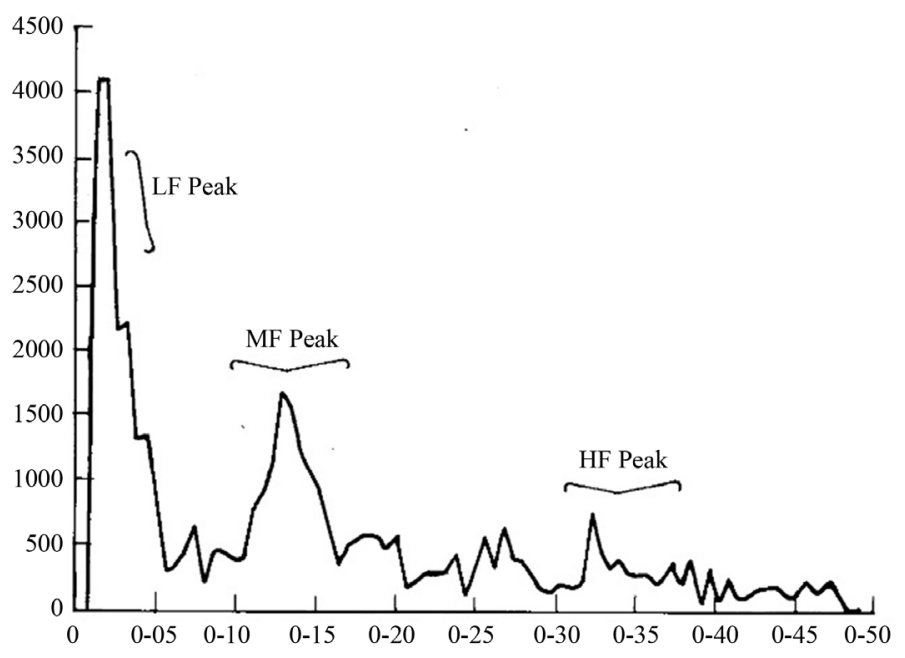

Figure 1. Typical PSD of a Vaatdosha dominance case. Here the peak is highest in the frequency range (LF) of Vaat. 
The following inferences for FR values for specific portion of the pulse waveform are statistically significant. Here, the Min and Max are of the FR values for $5^{\text {th }}$ to $45^{\text {th }}$ period of the pulse signal records.

For Healthy Male subjects:

(i) if Gunas $=(2,0,0)$, a sufficient condition for pulse strength $=$ "balwaan" is:

FR_min $=\sim 11.34$ and FR_max $=\sim 14.107$

(ii) if Gunas $=(2,0,0)$ a sufficient condition for pulse strength $=$ "ksheen" is:

FR_min $=\sim 9.34$ and FR_max $=\sim 11.27$

For Female subjects having Ischemic Heart Disease (IHD), Diabetes Mellitus (DM), and/or Hypertension (HT):

(i) if Gunas $=(1,0,0) \&$ pulse strength = "ksheen", a necessary and sufficient condition for pulse Depth $=$ "uttaan" is:

FR_min $=\sim 10.7$ and FR_max $=\sim 14.11$

(ii) if Gunas $=(1,0,0)$ \& nadi strength = "ksheen", a sufficient condition for pulse Depth = "gambheer" is:

FR_min $=\sim 13.06$ and FR_max $=\sim 18.04$

Our analysis for FR for the entire pulse record from $5^{\text {th }}$ period onwards, shows distinct range of values of FR for different intervals or categories of Feature values. We have also found distinct probability curves of FR for different categories (intervals) of several features that are regarded important in pulse-based diagnostic practices of Ayurveda. This indicates significant relationship between different Features and FR. We have analyzed male and female data separately for homogeneity of individual samples which was necessary for substantial ( $>90 \%)$ statistical confidence in the statistical estimates.

\subsubsection{Probability Function of FR}

The probability density function of FR estimated using the empirical plots of relative frequency in our data under different intervals/categories of certain features show distinct types or parameters. Significant results are shown in the Table 1 \& Table 2; some of these are also illustrated in Figure 2 and Figure 3. For other features intervals/categories that showed distinct probability distributions of FR, the samples were either too small or too heterogeneous.

Table 1. Significant results for the probability distribution of FR for data set of females.

\begin{tabular}{|c|c|c|c|}
\hline $\begin{array}{c}\text { Feature } \\
\text { Interval/Category }\end{array}$ & Approx. pdf of FR & $\begin{array}{c}\text { Feature } \\
\text { Interval/Category }\end{array}$ & Approx. pdf of FR \\
\hline Sys. BP $<100$ & Bi-modal: peaks at $\mathrm{FR}=10.5 \& \mathrm{FR}=14.5$ & Depth = uttaan & Truncated $N(11.5,9)$ \\
\hline Sys. BP $>110$ & Uniform $[8,16]$ & Depth = gambheer & Gamma: $k \approx 14 ; \alpha \approx 0.8$ \\
\hline $\begin{array}{l}\text { Guna_1 }<2 \\
\text { Guna_1 }=2 \\
\text { Guna_1 }>2\end{array}$ & $\begin{array}{l}\qquad \text { Gamma: } k \approx 14 ; \alpha \approx 0.84 \\
\text { Truncated Normal in the interval }[6.5,12.5) ; \\
\text { Cumulative of Uniform in }[12.5,17.5] \text { (Figure 2) } \\
\text { Gamma: } k \approx 12 ; \alpha \approx 0.9\end{array}$ & $\begin{array}{l}\text { Vaat } \leq 1 \\
\text { Vaat }>2\end{array}$ & $\begin{array}{c}\text { Gamma: } k \approx 18 ; \alpha \approx 0.6 \\
\text { Bi-modal: peaks at } \mathrm{FR}=11 \& \mathrm{FR}=16\end{array}$ \\
\hline Guna_2 $=0$ & Truncated $N(11.6,8.6)$ & Pitt $=1$ & Truncated $N(11.7,7.7)$ \\
\hline Guna_2 = 1 & Gamma in interval $[6.5,16.5)$; triangular in $[16.5,21.5]$ & Pitt $>1$ & Truncated $N(10.9,6.3)$ \\
\hline Bala $=$ balwaan & Gamma: $k \approx 13 ; \alpha \approx 0.9$ & $K a p h=0$ & Truncated $N(11.4,9.5)$ \\
\hline Bala = ksheen & Truncated $N(11.6,3.8)$ & Kaph $>0$ & Truncated $N(11,1.8)$ \\
\hline
\end{tabular}

Table 2. Significant results for the probability distribution of FR for data set of males.

\begin{tabular}{|c|c|c|c|}
\hline $\begin{array}{c}\text { Feature } \\
\text { Interval/Category }\end{array}$ & Approx. pdf of FR & $\begin{array}{c}\text { Feature } \\
\text { Interval/Category }\end{array}$ & Approx. pdf of FR \\
\hline $\begin{array}{l}\text { Sys. } \mathrm{BP}<110 \\
\text { Sys. } \mathrm{BP} \in[110,125] \\
\text { Sys. } \mathrm{BP}>125\end{array}$ & $\begin{array}{c}\text { Gamma: } k \approx 13 ; \alpha \approx 0.9 \\
\text { Bi-modal: peaks at } \mathrm{FR}=9 \& \mathrm{FR}=14 \text { (Figure 3) } \\
\text { Uniform }[8,17.5]\end{array}$ & $\begin{array}{c}\text { Depth = uttaan } \\
\text { Depth = gambheer }\end{array}$ & $\begin{array}{l}\text { Truncated } N(11.4,5.4) \\
\text { Gamma: } k \approx 10 ; \alpha \approx 1\end{array}$ \\
\hline $\begin{array}{l}\text { Guna_1 }<2 \\
\text { Guna_1 }=2 \\
\text { Guna_1 }>2\end{array}$ & $\begin{array}{c}\text { Gamma: } k \approx 14 ; \alpha \approx 0.85 \\
\text { Gamma: } k \approx 15 ; \alpha \approx 0.71 \\
\text { Cumulative of Uniforms in }[5,11) \&[11,19] .\end{array}$ & $\begin{array}{c}\text { Vat } \leq 1 \\
\text { Vat } \in[2,3] \\
\text { Vaat }>4\end{array}$ & $\begin{array}{l}\text { Gamma: } k \approx 13 ; \alpha \approx 0.9 \\
\text { Truncated } N(11,6.7) \\
\text { Uniform }[9.5,15.5]\end{array}$ \\
\hline $\begin{array}{l}\text { Guna_2 }=0 \\
\text { Guna_2 }=1\end{array}$ & $\begin{array}{c}\text { Truncated } N(11.3,7.7) \\
\text { Bi-modal: peaks at } \mathrm{FR}=10 \& \mathrm{FR}=16\end{array}$ & $\begin{array}{l}\text { Pitt } \leq 1 \\
\text { Pitt }>2\end{array}$ & $\begin{array}{c}\text { Truncated } N(11.3,7.4) \\
\text { Combination of two truncated } \\
\text { Normals in intervals }[6.5,11.5) \&[11.5,21.5]\end{array}$ \\
\hline $\begin{array}{c}\text { Bala = balwaan } \\
\text { Bala = ksheen }\end{array}$ & $\begin{array}{c}\text { Gamma: } k \approx 14 ; \alpha \approx 0.78 \\
\text { Truncated } N(12,8.6)\end{array}$ & $\begin{array}{l}\text { Kaph }=0 \\
\text { Kaph }>0\end{array}$ & $\begin{array}{c}\text { Uniform }[7.5,17.5] \\
\text { Truncated } N(11.4,4.5)\end{array}$ \\
\hline
\end{tabular}




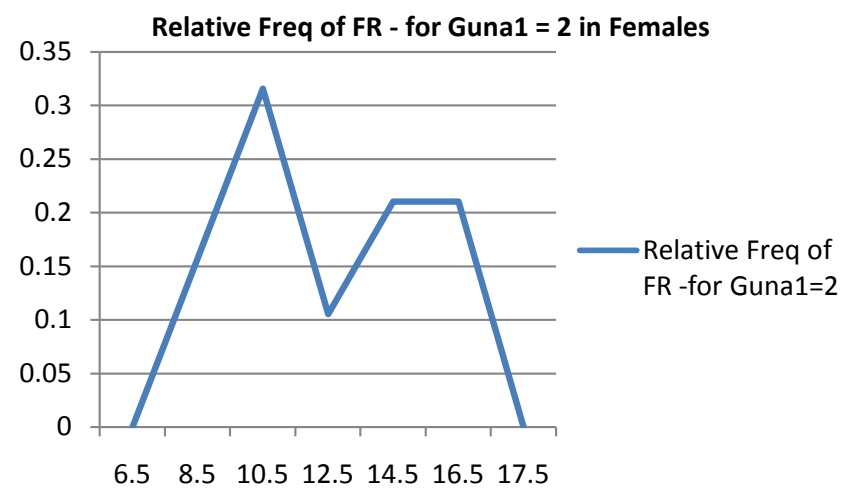

Figure 2. Relative frequency (shown along the vertical axis) of different values (intervals shown along the horizontal axis) of FR for pulse records of females who had Guna_1 $=2$.

Relative Freq of FR - for BP2 $(110,125]$ in Males

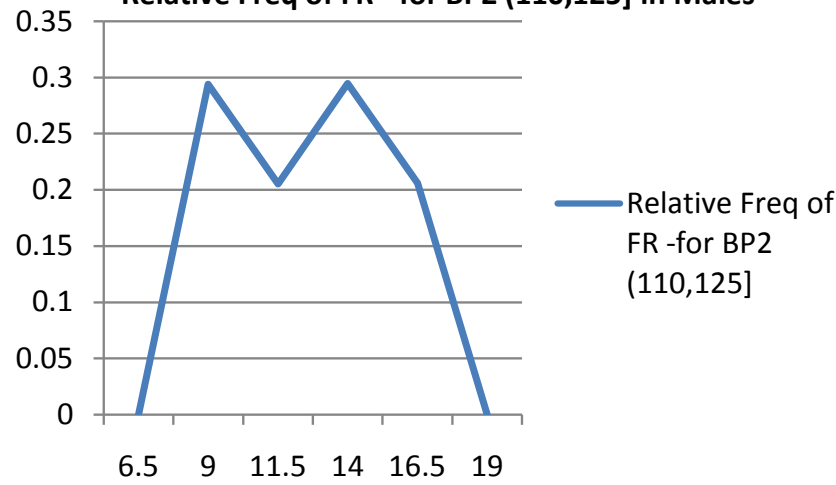

Figure 3. Relative frequency (shown along the vertical axis) of different values (intervals shown along the horizontal axis) of FR for pulse of males whose systolic BP was between 110 and 125 when their pulse signals were recorded.

\section{Discussion}

Renewed interest in the non-invasive, holistic diagnostic approach of pulse-examination followed in the traditional Chinese and Indian medicine has gained momentum with advanced instrumentation and digital recording (e.g. [12]-[14]). For pulse-based diagnosis/prognosis in Ayurveda - Traditional Indian medicine (TIM), patterns of pulse-velocity and pulse-movement are of crucial importance together with the pathological indicators in terms of doshas and several other features of pulse-sensation. Analysis of all these features was the key focus of our present research on pulse-examination.

Hierarchical clustering and multivariate statistical analysis has supported the relevance and utility of these TIM diagnostic features in classifying the healthy and diseased/pathological cases and also in differential diagnosis of the later [5] [10]. In the present study, Principal Component Analysis has shown the relative significance of specific features in classification and differential diagnosis of the healthy and diseased states. The doshas and features pertaining to pulse-strength (bala) are found most prominent followed by the features pulseproperties (gunas), etc. These findings further motivated us to analyze these features vis-à-vis pulse signals.

Recent research on pulse signals shows that spectral properties of pulse waveform are import in pulse-based diagnosis [2] [10] [14]. In an earlier project we had modeled pulse waveform as a function of doshas. Computational-simulation had shown definite role of the doshas in drastic changes in the morphology [4]. Our present study on pulse spectrum of real data also shows significant impact of the doshas on the spectral power density. Correlation studies with respect to large samples of specific pathological condition would be a welcome advancement of this research.

Our recently developed algorithm for accurate automatic detection of different patterns of pulse morphology 
and its variation over time as well the detection of consecutive patches of specific patterns/pattern-combination validated a crucial aspect of TIM practice of pulse-examination. It also made a notable, first ever contribution towards automated pulse-based diagnostic application [2].

As a particular spectral parameter, viz., the Fisher's likelihood Ratio (FR), was found important in our algorithm for computational detection of different patterns of pulse waveform, it was a natural extension to analyze the possible relation between FR and the TIM diagnostic features. The features called gunas pertaining to "pulse properties perceived through touch", by definition, "qualitatively" represent the pulse morphology. Interestingly, our results show a correlation between FR values for specific portion of the pulse (namely, between the $5^{\text {th }}$ and $45^{\text {th }}$ period) and the gunas.

Similar to other clinical/physiological/bio-chemical/bioelectrical features or parameters of the human mindbody system, pulse variation too is non-deterministic in nature. Whence, variation in FR should also be ideally analyzed as a random variable. We have therefore analyzed the empirical Probability Density Function ( $p d f$ ) of FR. (These functions were estimated by best fitting of different theoretical/standard probability density functions to the relative frequency curve obtained from the data.) The results show significant distinction in the form and/or parameters of the $p d f$ with respect different intervals or categories of several features.

It is interesting to note that non-standard or mixture of two standard distributions (e.g. in Figure 2 and Figure 3) are found in the cases that in terms of corresponding values or categories of the respective features would indicate-either (i) a pathological conditions, e.g. in the females having systolic BP $<100$; or (ii) different probability distributions of FR, i.e. different types and causes of variation in pulse morphology, e.g. in the group of females having Guna_1 = 2 or in the group of females having Guna_2 = 1; a priori, this is not an unusual condition so the group could practically represent large class of females, the diseased ones among which could be identified by taking into account some other features as well. Such types of non-standard " $p d f$ " indicate the need of analysis collectively with some other features.

The $p d f$ obtained as "Uniform [a, b]" shows that FR would take any value within [a, b] with equal probability. This practically means no inference on the pulse morphology patterns (and hence on diagnosis) in terms of the mode or other statistical parameter of FR, which means either the subject is healthy; e.g. in the group of Males having "Kaph" = 0; or there are different pathological conditions e.g. in the group of Males having systolic BP > 125 or in the groups of Males having Vaat > 4. In such a case, higher likelihood of distinct values of FR (i.e., the possibility of different patterns of pulse morphology) may be found by considering the specific range or category of the particular feature along with other feature(s) known as related with certain pathological condition. For example, high systolic BP together with higher body-weight and age would indicate risk of HT or cardiac problem, etc.

In the case of truncated Normal or Gamma, the most likely value (mode) of FR could be used for diagnostic inference in terms of pulse morphology provided the probability of this value is above certain threshold.

It should be noted that it was not our objective here to develop diagnostic rule or inference in terms of FR and/or any TIM feature. The aforesaid interpretation of the different forms of $p d f$ against respective feature intervals or categories is mainly to underline the additional importance and future applications of this approach.

For real application, the pdf should be estimated for FR of consecutive segments (that define pulse morphology patterns [2]) of the pulse record in different groups (of subjects) formed according to different intervals/ categories of features. Extended research on Multivariate Analysis incorporating all or several features jointly would be a landmark development towards comprehensive automated diagnostics.

As mentioned earlier, our objective and focus here was to analyze the relevance and importance, if any, of the TIM features for pulse-based diagnosis/prognosis. We have done it by analyzing the relation of these features with the spectral parameters of pulse that were found significant in recent instrumentation-based and computational research on pulse-based diagnosis/prognosis. Results show significant relation and hence validate the relevance, significance and utility of the TIM diagnostic features. In addition, this study also highlights new possibilities of development of comprehensive diagnostic techniques to authenticate, support and expand the traditional approach of pulse-examination in a consistent and objective manner.

\section{Acknowledgements}

The author thanks summer intern Aporupa Bose for assistance in probability-curve fitting. 


\section{References}

[1] Nawsupe, G. and Joshi, R.R. (2011) Modified Wavelet-Based Technique for Baseline Drift Removal and Diagnostic Scope of Spectral Energy of Radial Pulse Signal. International Journal of Biomedical Engineering and Technology, 6, 1-13. http://dx.doi.org/10.1504/IJBET.2011.040450

[2] Joshi, R.R., Nawsupe, G.B. and Wangikar, S.P. (2012) Automatic Detection of Pulse Morphology Patterns \& Cardiac Risks. Journal of Biomedical Science and Engineering, 5, 315-322. http://dx.doi.org/10.4236/jbise.2012.56041

[3] Joshi, R.R. (2004) A Biostatistical Approach Approach to Ayurveda-Quantifying the Tridosha. Journal of Alternative \& Complementary Medicine, 10, 879-889.

[4] Joshi, R.R. (2005) Diagnostics Using Computational Nadi Patterns. Mathematical and Computer Modeling, 41, 33-47. http://dx.doi.org/10.1016/j.mcm.2004.05.002

[5] Joshi, R.R., Wangikar, S.W. and Nawsupe, G. (2008) Instrumentation Based Pulse Diagnosis. DSS Technical Report, PRSG/Biomed/11-12, Ministry of Info. Tech., New Delhi.

[6] Diggle, P. and Fisher, N.I. (1991) Nonparametric Comparison of Cumulative Periodograms. Applied Statistics, 40, 423-434. http://dx.doi.org/10.2307/2347522

[7] Hankey, A. (2001) Ayurvedic Physiology and Etiology: Ayurvedo Amritanaam. The Doshas and Their Functioning in Terms of Contemporary Biology and Physical Chemistry. The Journal of Alternative and Complementary Medicine, 7, 388-396. http://dx.doi.org/10.1089/10755530152639792

[8] Patwardhan, B., et al. (2005) Classification of Human Population Based on HLA Gene and the Concept of Prakrati in Ayruveda. Journal of Alternative \& Complementary Medicine, 11, 349-353.

[9] Hankey, A. (2010) Establishing the Scientific Validity of Doshas, Subdoshas and Dosha Prakritis. Ancient Science of Life, 29, 7-21.

[10] Joshi, R.R., Nawsupe, G.B. and Wangikar, S.P. (2012) Ayurvedic Nadi Parikshanki Adhunik Vaigyanic Waidhata: Chikitsakiya Ankadonka Sankhyikiya aur Sanganatmak Vishleshana (Statistical \& Computational Analysis of Ayurvedic Pulse Diagnostic Data). JIGYASA (special issue), 27 \& 28, 16-25.

[11] Everitt, B.S. and Dunn, G. (2001) Applied Multivariate Data Analysis. 2nd Edition, Hodder Arnold, London. http://dx.doi.org/10.1002/9781118887486

[12] Abhinav, Sareen, M., Kumar, M., Anand, S., Salhan, A. and Santosh, J. (2009) NadiYantra: A Robust System Design to Capture Signals from the Radial Artery for Assessment of the Autonomic Nervous System Non-Invasively. Journal of Biomedical Science \& Engineering, 2, 471-479. http://dx.doi.org/10.4236/jbise.2009.27068

[13] Karamchandani, S., Merchant, S.N., Desai, U.B. and Jindal, G.D. (2010) Application of Crisp and Fuzzy Clustering Algorithms for Identification of Hidden Patterns from Plethysmographic Observations on the Radial Pulse. Proceedings of Annual International Conference of the IEEE Engineering in Medical \& Biolology Society, 1, 3978-3981.

[14] O’Ruke, M.F. (2004) Pulse Waveform Analysis and Arterial Stiffness: Realism Can Replace Evangelism and Skepticism. Journal of Hypertension, 22, 1633-1634. http://dx.doi.org/10.1097/01.hjh.0000125473.35523.3f 
Scientific Research Publishing (SCIRP) is one of the largest Open Access journal publishers. It is currently publishing more than 200 open access, online, peer-reviewed journals covering a wide range of academic disciplines. SCIRP serves the worldwide academic communities and contributes to the progress and application of science with its publication.

Other selected journals from SCIRP are listed as below. Submit your manuscript to us via either submit@scirp.org or Online Submission Portal.
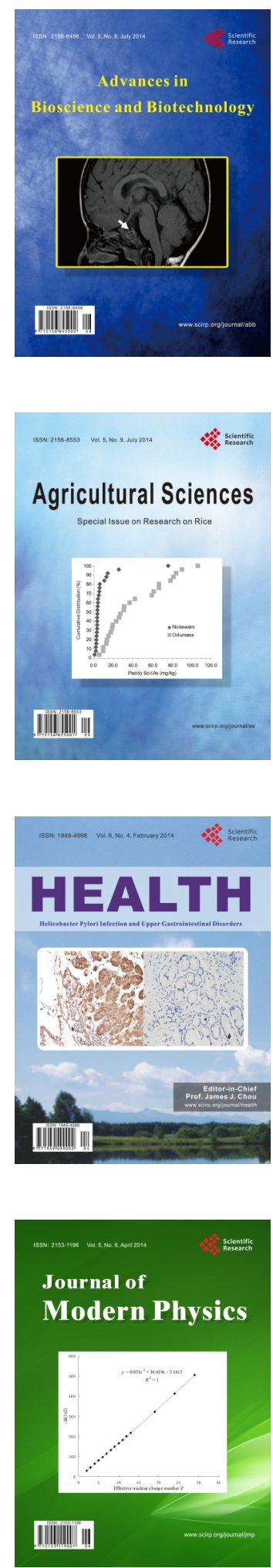
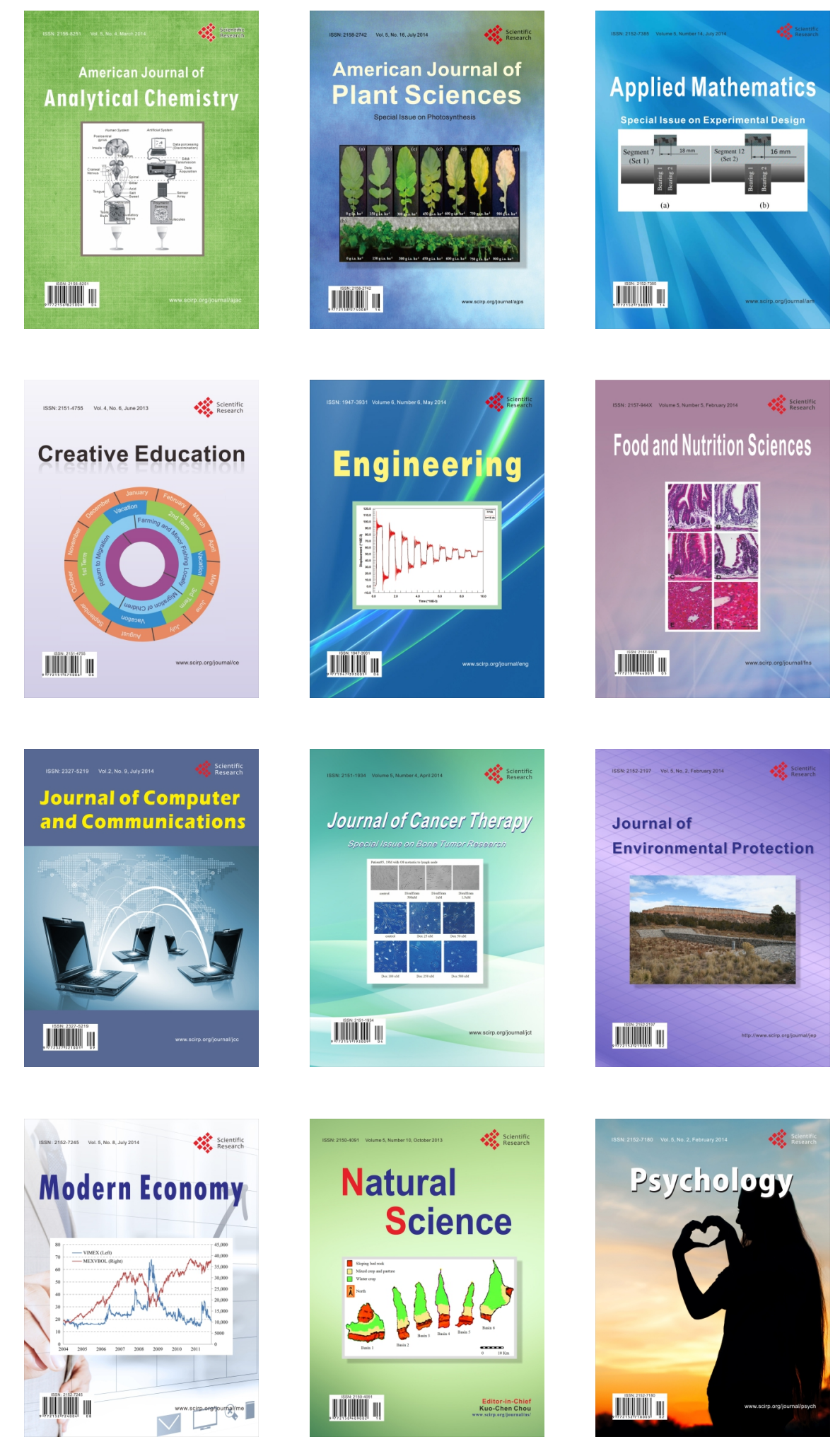